\title{
The Formulation of Minimal Clinically Important Differences in the Quality of Life Instrument for Patients with Chronic Renal Failure (QLICD-CRF) Based on Combinations of Anchor-Based and Distribution-Based Methods
}

\section{Xinping Huang}

Dongguan Enghth People's Hospital https://orcid.org/0000-0002-1024-7862

\section{Zheng Yang}

Guangdong Medical University

\section{Min Weng}

The First Affiliated Hospital of Kunming Medical University

\section{Xuejin Fan}

Dongguan Eighth People's Hospital

\section{Qingan Xu}

Guangdong Medical University

Chonghua Wan ( $\square$ wanchh@hotmail.com )

Guangdong Medical University

\section{Research article}

Keywords: Chronic renal failure, Quality of life, Minimal clinically important difference (MCID)

Posted Date: September 21st, 2020

DOI: https://doi.org/10.21203/rs.3.rs-48375/v1

License: (c) (1) This work is licensed under a Creative Commons Attribution 4.0 International License.

Read Full License 


\section{The formulation of minimal clinically important differences in the Quality}

\section{of Life Instrument for Patients with Chronic Renal Failure (QLICD-CRF)}

\section{based on combinations of anchor-based and distribution-based methods}

Xinping Huang ${ }^{1}$, Zheng Yang ${ }^{2}$, Min Weng ${ }^{3}$, Xuejin Fan ${ }^{1}$, Qingan Xu ${ }^{4}$, Chonghua Wan ${ }^{4}$

${ }^{1}$ Xinping Huang, Dongguan Enghth' Hospital, Dongguan 523325, No 68, West Xihu 3rd Road, Dongguan city, Guangdong

Province, CHINA; sunnyhuang2015@163.com

${ }^{2}$ School of Public Health, Guangdong Medical University, Dongguan 523808, CHINA;

yangzheng63@hotmail.com

${ }^{3}$ The First Affiliated Hospital of Kunming Medical University, 650100, CHINA; wengmincat@yahoo.com.cn

${ }^{1}$ Xuejin Fan, Dongguan Enghth’ Hospital, Dongguan 523325, CHINA; Boaifanxuejin@ 163.com

${ }^{4}$ Qingan Xu, School of Humanities and Management, Research Center for Quality of Life and Applied Psychology, Guangdong Medical University, Dongguan 523808, CHINA; qingan_xu@163.com

${ }^{4}$ Chonghua Wan, School of Humanities and Management, Research Center for Quality of Life and Applied Psychology, Guangdong Medical University, Dongguan 523808, CHINA; wanchh@ hotmail.com

Grants: Supported by the National Natural Science Foundation of China $(71373058,81460519)$ and the Science and Technological Planning Program of Guangdong Province (2013B021800074).

\# Xinping Huang and Zheng Yang are co-first authors with equal contributions.

${ }^{*}$ Correspondence to: Chonghua Wan (Email: wanchh@hotmail.com.) 
Abstract:

Background Chronic renal failure is global problem and represents a major cause of morbidity and mortality. Studies of quality of life in patients with chronic renal failure can be helpful to improve patient survival rates. The minimal clinical important difference (MCID) is a score that reflects the effect of patient treatment or nursing care. Doctors or researchers use this factor to assess the validity of results, and this information reflects the combined importance of the patient's opinion and the doctor's explanation.

Methods Patients diagnosed with chronic renal failure and able to read and understand the questionnaires at the First Affiliated Hospital of Kunming Medical University were included in this study and completed the questionnaires independently. Using the anchor-based method and the distribution-based method, the MCIDs of the QLICD-CRF were calculated based on the data from 164 valid questionnaires at the time of admission and 111 valid questionnaires at discharge. the anchor-based method, whereas the results calculated based on effect size (ES), standard error of measurement (SEM) and reliable change index (RCI) were referred to as MCIDs based on the distribution-based method. Then, the final results were calculated according to the weight of the calculation obtained using several methods.

Results MCIDs of the physical domain (PHD), psychological domain (PSD), social domain (SOD), core/general domain

Conclusion Calculations of MCIDs of QLICD-CRF scores could compensate for insufficient $P$-values; thus, the interpretation of the outcomes in quality of life studies could be more objective and specific.

\section{Background}

45 Chronic renal failure is global problem and represents major cause of morbidity and mortality. Mortality 
caused by chronic renal failure increased significantly from the 27th position in 1990 to 18th position in $2010^{[1]}$. Currently, more than 2 million people worldwide receive treatment in form of dialysis or a kidney transplant to stay alive, representing only $10 \%$ of people who actually need treatment to live ${ }^{[2]}$. Study of the quality of life of patients with chronic renal failure can be helpful to improve survival rates. Thus, numerous scales were developed, such as the Kidney Disease Quality of Life instrument (KDQOL), the Kidney Transplant Questionnaire, and the choice health experience questionnaire. However, these scales do not completely reflect the background of Chinese culture.

To adapt these scales to Chinese culture, we developed the quality of life measurement scale (QLICD-CRF V2.0) for patients with chronic renal failure through repeated expert discussions and combinations of various statistical methods ${ }^{[3-7]}$. The QLICD-CRF V2.0 consists of two parts: the generic module ${ }^{[3-4]}$ and the specific module ${ }^{[5]}$. The generic module includes physical, psychological, and social domains mainly aimed at the general effects of chronic diseases, and the specific module was developed to assess the specific effects of chronic renal failure. Assessment of reliability and validity of the scale were reported in another $\operatorname{article}^{[6-7]}$.

Quality of life scores are used to evaluate subjective feelings of patients. What is the guiding significance of these scores in the clinic? We should determine the effective threshold of the fraction change and other traditional factors, such as reliability, validity, and reaction. Statistically, the $P$-value will be less than the test level if sample size is sufficiently large. However, to what extent do changes in quality of life score changes indicate clinical significance? This value can be measured using the minimal important difference (MID), minimal clinically important difference (MCID), minimal clinically important changes (MCIC), clinically meaningful changes (CMC), or clinically significant changes (CSC). In this article, we refer to this parameter as minimal clinically important difference (MCID), which is a score change that reflects the 
effect of the patient's treatment or nursing support ${ }^{[8]}$. Doctors and researchers use this information to judge the validity of their results, and this information importantly combines the patient's opinion with the doctor's explanation.

Current research on MCID is mainly reported from studies abroad ${ }^{[9-18]}$. Studies on MCID in China are limited $^{[19-20]}$, and most of these studies involve traditional Chinese medicine. Various methods, such as the anchor-based approach, distributed-based approach, expert opinion and literature analysis, are used to calculate the MCID, but there is no "gold standard". Each method has disadvantages. Most current studies typically use one of these methods. The purpose of our study was to determine MCID of QLICD-CRF using a combination of methods. In our study, MCID was determined according to the results of QLICD-CRF scores of patients with chronic renal failure.

\section{Methods}

\subsection{Participants}

The subjects were patients with chronic renal failure who can read and write. These patients were treated at the First Affiliated Hospital of Kunming Medical University. We relied on clinicians to recruit participants who participated in the study after providing informed consent. The Institutional Review Board of the investigators' institutions approved the study protocol and informed consent form.

Inclusion criteria: (1) clinically diagnosed with chronic renal failure, (2) at least primary and secondary school-level education, (3) volunteer to participate in the evaluation, and (4) able to read and write.

Exclusion criteria: (1) illiterate, (2) patients who were mentally inarticulate or unable to express his inner feelings clearly, and (3) critically ill patients.

\subsection{Investigation instruments}


We used the QLICD-CRF and SF-36 to investigate patients with chronic renal failure ${ }^{[7]}$. The anchor was selected from items from SF-36.

Our QOL team developed the QLICD-CRF. A module approach was used for the development of the chronic renal failure scale of the system of quality of life instruments for chronic diseases (QLICD-CRF V2.0), including the general module (QLICD-GM) for all types of chronic diseases and a disease-specific module for patients with chronic renal failure. The final 28-item scale (QLICD-GM V2.0) can be classified into 3 domains and 9 facets. The physical domain (PHD) includes 9 items coded from GPH1 to GPH9, which are divided into 3 facets of Basic Physiological Functions (BPF), Energy and Discomfort (EAD), and Independence (IND). The psychological domain (PSD) contains 11 items coded from GPS1 to GPS11, which are divided into 3 facets of Cognition (COG), Will and Personality (WIP), and Emotion (EMO). The social domain (SOD) comprises 8 items coded from GSO1 to GSO8, which are divided into 3 facets of Interpersonal Communication (INC), Social Support and Security (SSS), and Social Role (SOR). The disease-specific module for patients with chronic renal failure includes 10 items, which are divided into 3 facets: Respiratory and Circulation System Symptom (RCS), Musculoskeletal and Derma Symptom (MDS), Abnormal Stool and Urination (ASU).

The Chinese version of SF-36 ${ }^{[21]}$, which comprises eight domains, including Physical Function (PF), Role-Physical (RP), Bodily Pain (BP), General Health (GH), Vitality (VT), Social Function (SF), Role-Emotional (RE) and Mental Health (MH), was also used to obtain data to assess the criterion-related validity of the QLICD-CRF as well as convergent and discriminant validity.

\subsection{Methods}

The participating investigators (medical postgraduates) explained the purpose and the scales to qualified 
111 patients and obtained informed consent from patients who agreed to participate in the study. The patients 112 were required to complete the scales (the QLICD-CRF and SF-36) independently on the day of hospital 113 admission and once again at the day of discharge.

114 We calculated some indexes using the anchor-based method and the distribution based-method. Then, the 115 final MCID values were calculated according to the weights of the calculation of several methods.

The anchor-based method: The anchor was one of the items in SF-36 (item 10d) expressed as "My health

117 is excellent". Possible answers include the following: 1, Definitely True; 2, Mostly True; 3, Do not Know; 4, Mostly False; 5, Definitely False. The data were compared before and after treatment using the following 119 steps:

(1) Patients completed the two questionnaires twice at the time of admission and discharge.

(2) Patients who report a difference of one level in responses are removed from analysis. For example, patient A selects the option "Definitely True" first. After treatment, he/she chose "Mostly True". Thus, patient A was included in the analyses using the anchor-based method. Similarly, patient B chose the option

"Definitely False" on the first questionnaire. After treatment, he/she selected "Mostly False". Thus, patient B was also included in the analyses using the anchor-based method. Thus, when the absolute value of the difference in the option number selected before and after treatments was "1", the patient's response was included.

(3) The absolute value of the difference of quality of life was employed to calculate the score of each patient who was selected. If the absolute value of the difference exhibited a normal distribution, the mean value was used as MCID. If a skewed distribution was observed, the median was reported as the MCID. In our study, responses from 39 patients were analysed using the anchor-based method. The absolute values of the differences exhibited a skewed distribution, so the median was reported. 
The distribution based-method: The distribution based-method requires the collection of data both before and after treatment. There are three indexes, including effect size (ES), standard error of measurement (SEM) and reliable change index (RCI), which employ the following questions $\mathrm{MCID}=\mathrm{ES} \times \mathrm{SD}_{\text {before }}$, $\mathrm{MCID}=1 \times \mathrm{SEM}^{[22]}$, and $\mathrm{MCID}=1 \times \mathrm{RCI}$, respectively. Computational formulas are provided in Table 1.

\begin{tabular}{lll}
\hline Index & Formula & Explanation \\
\hline
\end{tabular}

ES: effect size; mean ${ }_{\text {before: }}$ standardized mean before ES $\quad \mathrm{ES}=\frac{\text { mean }_{\text {before }}-\text { mean }_{\text {after }}}{S D_{\text {before }}}$ treatment: mean $_{\text {after: }}$ standardized mean after treatment; $\mathrm{SD}_{\text {before }}$; standard deviation before treatment SEM: standard error of measurement; $\sigma_{\mathrm{x}}$ : standard SEM $\mathrm{SEM}=\sigma_{\mathrm{x}} \sqrt{1-\mathrm{r}_{\mathrm{tt}}}$ deviation of the quality of life measurement; ; $\mathrm{r}_{\mathrm{tt}}$ : reliability of scale

$$
\begin{gathered}
\mathrm{RCI}= \\
\mathrm{RCI} \frac{\text { mean }_{\text {before }}-\text { mean }_{\text {after }}}{\sqrt{2 S E M^{2}}} \quad \text { RCI: reliable change index }
\end{gathered}
$$

Weighting method: The MCID is comprehensively considered on the basis of the weight of results of different methods. By comparing every result from different methods with the "Mean of the four results", a weight was given based on the deviation from the mean. We gave a higher weight value to the results of MCID (M) and ES because these methods had higher degrees of acceptance. 
144 In total, 164 patients were investigated in our study. All of the patients completed the two scales on

145 admission, but only 111 completed the two scales at the time of discharge. The socio-demographic

146 characteristics of the sample are presented in Table 2. The average patient age was (45.57士14.94) years.

147 There were more males than females. Most of the patients were of Han nationality, and most of had

148 graduated from high school or technical secondary school or even less. The proportion of farmers in the

149 vocational structure was relatively large. Regarding medical insurance, urban and rural workers comprised

150 the largest demographic with medical insurance.

Table 2 The characteristics of 164 patients with chronic renal failure

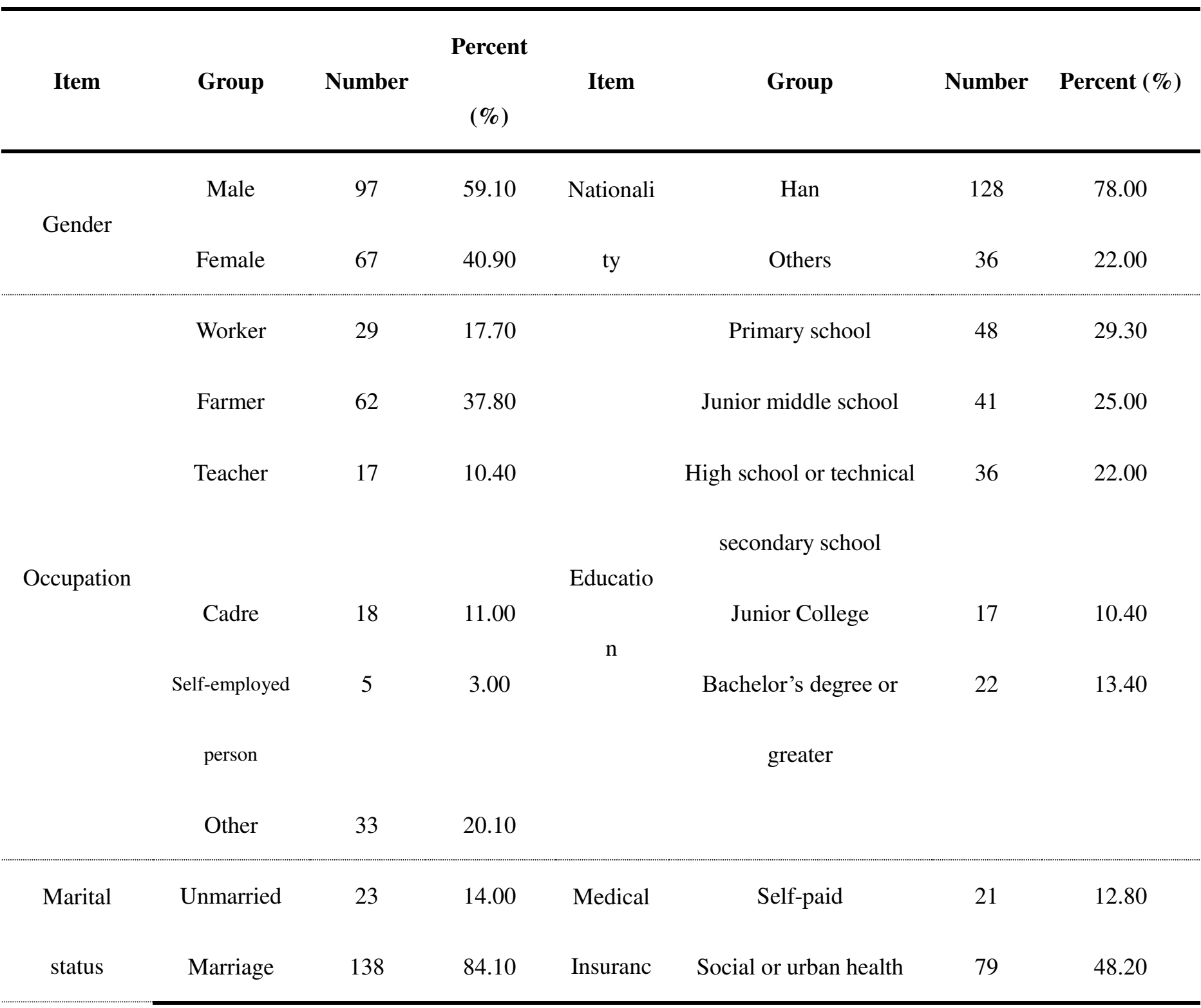


e

$\begin{array}{cccccr}\text { Divorce } & 2 & 1.20 & \text { Commercial insurance } & 1 & 0.60 \\ \text { Widowed } & 1 & 0.60 & \text { Rural cooperative } & 57 & 34.80 \\ & & \text { medical service } & \end{array}$

care

\begin{tabular}{cccc} 
& Poor & 84 & 51.20 \\
Family & Moderate & 75 & 45.70 \\
& & & 3.00 \\
\hline
\end{tabular}

152

\subsection{Calculation of MCID based on the anchor method}

154 In our study, the anchor was the item in SF-36 (10d) that was described as "My health is excellent". In total,

15539 patients were included in analyses using the anchor method, and the results are provided in Table 3 in 156 detail.

Table 3 Calculation of MCID based on the anchor method

\begin{tabular}{lcc}
\multicolumn{1}{c}{ Domains } & Numbers & MCID (M) \\
\hline Physical domain (PHD) & 38 & $\mathbf{1 2 . 5 0}$ \\
Psychological domain (PSD) & 38 & $\mathbf{9 . 0 9}$ \\
Social domain (SOD) & 38 & $\mathbf{9 . 3 8}$ \\
Core/General domain (CGD) & 37 & $\mathbf{6 . 9 0}$ \\
Specific domain (SPD) & 37 & $\mathbf{4 . 0 0}$ \\
Total (TOT) & 35 & $\mathbf{6 . 8 5}$ \\
\hline
\end{tabular}

159 The distribution based-method used ES, SEM, and RCI to calculate MCID. The results are presented in Tables 3 


\begin{tabular}{lccccc}
\hline \multicolumn{1}{c}{ Domains } & Number & $\sigma_{\mathrm{x}}$ & $\mathrm{r}_{\mathrm{tt}}$ & SEM & RCI \\
\hline Physical domain (PHD) & 111 & 17.68 & 0.70 & 9.68 & 13.69 \\
Psychological domain (PSD) & 111 & 19.99 & 0.75 & 10.00 & 14.14 \\
Social domain (SOD) & 111 & 16.42 & 0.70 & 8.99 & 12.71 \\
Core/General domain (CGD) & 111 & 15.81 & 0.76 & 7.75 & 10.96 \\
Specific domain (SPD) & 111 & 18.27 & 0.67 & 10.50 & 14.85 \\
Total (TOT) & 111 & 15.77 & 0.77 & 7.56 & 10.69 \\
\hline
\end{tabular}

\begin{tabular}{lcccc}
\hline \multicolumn{1}{c}{ Domain } & SD $_{\text {before }}$ & ES (0.2) & ES (0.5) & ES (0.8) \\
\hline Physical domain (PHD) & 17.37 & 3.47 & $\mathbf{8 . 6 9}$ & 13.90 \\
Psychological domain (PSD) & 20.32 & 4.06 & $\mathbf{1 0 . 1 6}$ & 16.26 \\
Social domain (SOD) & 16.56 & 3.31 & $\mathbf{8 . 2 8}$ & 13.25 \\
Core/General domain (CGD) & 15.87 & 3.17 & $\mathbf{7 . 9 4}$ & 12.70 \\
Specific domain (SPD) & 19.32 & 3.86 & $\mathbf{9 . 6 6}$ & 15.46 \\
Total (TOT) & 16.19 & 3.24 & $\mathbf{8 . 1 0}$ & 12.95 \\
\hline
\end{tabular}

164 


\begin{tabular}{lccccc}
\hline \multicolumn{1}{c}{ Domains } & MCID (M) & SEM & ES (0.5) & RCI & methods \\
\hline Physical domain (PHD) & $\mathbf{1 2 . 5 0}$ & 9.68 & $\mathbf{8 . 6 9}$ & 13.69 & 11.14 \\
Psychological domain (PSD) & $\mathbf{9 . 0 9}$ & 10.00 & $\mathbf{1 0 . 1 6}$ & 14.14 & 10.85 \\
Social domain (SOD) & $\mathbf{9 . 3 8}$ & 8.99 & $\mathbf{8 . 2 8}$ & 12.71 & 9.84 \\
Core/General domain (CGD) & $\mathbf{6 . 9 0}$ & 7.75 & $\mathbf{7 . 9 4}$ & 10.96 & 8.39 \\
Specific domain (SPD) & $\mathbf{4 . 0 0}$ & 10.50 & $\mathbf{9 . 6 6}$ & 14.85 & 9.75 \\
Total (TOT) & $\mathbf{6 . 8 5}$ & 7.56 & $\mathbf{8 . 1 0}$ & 10.69 & 8.30 \\
\hline
\end{tabular}

\subsection{Weight method}

Every result obtained from different methods was compared with the "Mean of the four methods", and a weight was given based on the deviation from the mean. We gave a higher weight value to the results of MCID (M) and ES because these methods have higher degree of acceptance.

Table 6 Weights of the four methods and MCID calculated using the weight method

\begin{tabular}{|c|c|c|c|c|c|c|}
\hline \multirow{3}{*}{ Domains } & MCID & \multirow{3}{*}{ SEM } & \multirow{3}{*}{ ES (0.5) } & \multirow{3}{*}{$\mathrm{RCI}$} & \multirow{3}{*}{$\begin{array}{c}\text { MCID } \\
\text { (Weighted) }\end{array}$} & \multirow{3}{*}{$\begin{array}{l}\text { Take an } \\
\text { integer }\end{array}$} \\
\hline & & & & & & \\
\hline & (M) & & & & & \\
\hline Physical domain (PHD) & 0.40 & 0.35 & 0.15 & 0.10 & 10.15 & 10 \\
\hline Psychological domain (PSD) & 0.15 & 0.35 & 0.45 & 0.05 & 9.36 & 9 \\
\hline Social domain (SOD) & 0.45 & 0.40 & 0.10 & 0.05 & 8.38 & 8 \\
\hline Core/General domain (CGD) & 0.10 & 0.40 & 0.45 & 0.05 & 7.24 & 7 \\
\hline
\end{tabular}




\begin{tabular}{lcccccc}
\hline Specific domain (SPD) & 0.05 & 0.45 & 0.50 & 0 & 8.27 & 8 \\
Total (TOT) & 0.15 & 0.35 & 0.45 & 0.05 & 7.22 & 7 \\
\hline
\end{tabular}

According to Table 6, the mean of these domains was the weight of the corresponding method result. Thus, the weight of MCID (M), SEM, ES, and RCI were $21.67 \%, 38.33 \%, 35 \%$, and $5 \%$, respectively.

The results of the different methods in Table 5 were multiplied by the respective weights, so we obtained the MCID of every domain. For example, the MCID of PHD is calculated as follows: $12.50 * 21.67 \%+9.68 * 38.33 \%+8.69 * 35 \%+13.69 * 5 \%=10.15$.

\section{Discussion}

The minimal clinically important difference score (MCID) was developed ${ }^{23]}$ in 1989 in an attempt to deal with the problem that analysis of a PROM instrument measuring change after intervention in some cases may show statistical significance without any clinical relevance. Doctors and researchers use this information to judge the validity of their results, and this information importantly includes the patient's opinion and the doctor's explanation. The quality of life measurement scale (QLICD-CRF V2.0) for patients with chronic renal failure exhibits good reliability and validity ${ }^{[3-7]}$. This scale is ideally suited for

Chinese patients. In our study, no large differences were noted among the four results from the anchor-based method and the distribution based-method. MCID (M) values obtained using the anchor-based method were greater than values obtained from other methods. This result is potentially attributed to the fact that the sample size was small, but it does not indicate that the results make no sense. 
ulcers, 27 with limb reconstruction, 25 with irritable bowel syndrome, and 25 with COPD. Two disadvantages of the anchor-based method are noted. First, it was difficult to choose a representative anchor; second, the sample size required to calculate MCID was much smaller than the sample size investigated in this study. Researchers must include more participants to ensure that the sample is sufficient and representative. Thus, the research costs were increased.

Effect size (ES) can be used to calculate MCID. ES calculations are simple, and nonprofessional individuals can easily understand the method. Cohen ${ }^{[20]}$ argued that an effect size (ES) between $0.2-0.49 \%$ was small, $0.5-0.79 \%$ was medium, and greater than or equal to 0.8 was large. In our study, an effect size of up to 0.5 is accepted by most people. An effect size of 0.2 indicates the smallest score change and this finding was not very clinical significant. To allow the results to be recognized by many scholars and clinicians and make the results more consistent with clinical practice, we chose the score changes of $\mathrm{ES}=0.5$ as significant MCID of domains and total scores in QLICD-CRF.

SEM and RCI do not depend on the sample size because the two indexes estimate the MCID through the baseline standard deviation and reliability coefficient. The MCID obtained based on ES is more accepted, and the calculation is also more convenient.

This study comprehensively evaluated the state of patients by combining patients' subjective feelings and clinical indicators. This information could be useful to improve patient satisfaction and the doctor-patient relationship. The current study primarily used a single anchor-based method to calculate the MCID of different scales; then, an ROC curve was used to identify the most effective scale ${ }^{[25-29]}$. A few studies have used multiple methods to calculate MCID. For example, Shulman MA used three distribution-based methods (0.3 multiplied by SD, standard error of the measurement, and 5\% range) and two anchor-based methods (anchored to two patient-rated health status questions and separately to unplanned hospital 
readmission) to estimate the MCID for the World Health Organization Disability Assessment Schedule 2.0

218 score converted to a percentage scale ${ }^{[30]}$. Toussaint A used effect sizes (ES) and standardized response

219 means (SRM) of pre- and post-GAD-7 mean changes to calculate MCID for the Hamilton Rating Scale for

220 Depression (HRSD-24) ${ }^{[31]}$. In this study, multiple methods were used to calculate the comprehensive MCID

221 according to the weights, and the advantages and disadvantages of various methods were considered.

222 Our study has also several limitations. First, the sample size was smaller compared with some other studies,

223 especially for the anchor-based method. Second, the subjects in this study were selected from the inpatient

224 population at hospitals. Additional studies are needed to assess the practicability of MCID evaluation in

225 other settings and populations, such as outpatients at a local clinic.

\section{Conclusions}

Every method has its limitations and advantages. The results of various methods are considered in the weight method, so we recommend weight method to increase the acceptance of results. Finally, the following MCIDs of QLICD-CRF are obtained: PHD 10, PSD 9, SOD 8, CGD 7, SPD 8, and TOT 7.

\section{Additional files}

\section{Abbreviations}

minimal clinically important differences (MCID)

Chronic Diseases (QLICD-CRF).

Kidney Disease Quality of Life instrument (KDQOL)

Physical domain (PHD) 
Energy and Discomfort (EAD)

241 Independence (IND)

242 Psychological domain (PSD)

243 Will and Personality (WIP)

244 Emotion (EMO)

245 Social domain (SOD)

246 Interpersonal Communication (INC)

247 Social Support and Security (SSS)

248 Social Role (SOR)

249 Respiratory and circulation system symptom (RCS)

250 Musculoskeletal and Derma symptom (MDS)

251 Abnormal stool and urination (ASU)

\section{Declarations}

253 -Ethics approval and consent to participate:Our study was submitted to and approved by The ethics

254 committee of Kunming Medical College (currently known as Kunming Medical University) institutional ethics committee and approved the study.the consent we obtained from study participants was written.

- Consent for publication: Not applicable.

257 - Availability of data and materials:If anyone would like to access data related to the study, please

258 contact corresponding author Chonghua Wan, email: wanchh@ hotmail.com.

- Competing interests: The authors declare that they have no competing interests. 
- Funding:This study was supported by the National Natural Science Foundation of China (71373058 and 81460519) and the Science and Technological Planning Program of Guangdong Province (2013B021800074). The funding bodies provided funds to support project development. The grant recipient (Chonghua Wan) designed the study, performed the data collection and data analyses, and extensively revised the manuscript.

- Authors' Contributions:XPH, ZY, and CHW designed the study. XPH, MW, XJF and CHW performed the data collection. XPH, ZY, QAX and CHW performed data analyses. XPH drafted the manuscript. ZY and CHW extensively revised the manuscript. All authors contributed to data interpretation. All authors have read and approved the final manuscript.

- Acknowledgements:In this research project, we received substantial assistance from staffs of the First Affiliated Hospital of Kunming Medical University . . We sincerely acknowledge all the support.

\section{References:}

1. Rame Miftari,Adem Nura, Valdete Topçiu-Shufta,et al.Impact of Gate 99mTc DTPA GFR, Serum Creatinine and Urea in Diagnosis of Patients with Chronic Kidney Failure[J]Acta Inform Med. 2017 Jun; 25(2): $99-102$.

2. Couser WG, Remuzzi G, Mendis S, Tonelli M. The contribution of chronic kidney disease to the global burden of major non communicable diseases. Kidney Int. 2011;80(12):1258 - 70. 
3.WAN Chong-Hua ,GAO Li, LI Xiao-Mei,, et al. Development of the Gener al Module for the System of Quality of Life Instruments for Patients with Chronic Disease: Items selection and structure of the gener al module. [J]. Chinese Mental Health Journal, 2005, 12 (1) : 723-726.

4.PAN Hai-yan,YANG Zheng,WAN Chong-Hua,etal.Evaluation of the General of Quality of Life Instruments for Chronic Disease[J].Chinese General Practice,2012,15（4A）:1101-1107.

5. WENG Min,GAO Sai,HUANG Xin-ping,etal.Item selection of the development of the quality of life Instrument for maintenance hemodialysis patients [J].International Journal of Transplantation and Hemopurification,2009,7（6）:25-28.

6. YANG Zheng, HUANG Xin-ping, WENG Min, etal.Validity evaluation of the quality of life instruments for chronic diseases-chronic renal failure scale (QLICD-CRF)[J].Journal of Guangdong Medical College, 2014, $32(3): 401-404$.

7. Yang Zheng, Huang Xinping, Wan Chonghua, et al. Reliability Evaluation of the Quality of Life Instrument for Patients with Chronic Renal Failure ( QLICD-C R F) based on Classical Test Theory and Generalizability Theory[J]. Chinese Journal of Health Statistics ,2015,32(02):224-226.

8.Kathleen W. Wyrwich, Bullinger M,et al. Estimating clinically significant differences in quality of life outcomes. [J]Qual Life Res ,2005(14): 285 - 295.

9.Norman, GR, Sloan, JA, and Wyrwich, KW. Interpretation of changes in health related quality of life: The remarkable universality of half a standard deviation. [J]Med Care 2003; 41(5): 582 - 592.

10.Stephen J. Walters, John E, et al. Comparison of the minimally important difference for two health state utility measures: EQ-5D and SF-6D. [J] Quality of Life Research (2005)14: 1523 - 1532.

11. Ringash J, Bezjak A, Sullivan B, et al .Interpreting differences in quality of life: The FACT-H\&N in laryngeal cancer patients. [J] Quality of Life Research 2004 ,13: 725 - 733. 

VAS scores in cancer. [J] Health and Quality of Life Outcomes 2007, 5:70 important difference in patients with type 2 diabetes Health and [J]Quality of Life Outcomes 2009, 7:91. 14.Osoba D, Rodrigues G, Myles J, et al. Interpreting the significance of changes in health-related quality of life scores. [J] J Clin Oncol 1998; 16: 139 - 144. Quality of life Index (DLQI): Results from patients with chronic idiopathic urticaria. [J] Health and Quality of Life Outcomes 2005, 3:36.

16. Puhan M, Frey M, Büchi S, e tal. The minimal important difference of the hospital anxiety and depression scale in patients with chronic obstructive pulmonary disease[J]. Health and Quality of Life Outcomes 2008, 6:46.

17.Yeung MT,Wong WP,Huang K,et al. Minimal clinically important difference for 6-minute walk distance in singaporean adults with chronic respiratory diseases[J]. Chinese Journal of Rehabilitation Medicine.2015,25(1):26-33.

18.Reddy VK,Parker SL,Patrawala SA,et al. Microvascular decompression for classic trigeminal neuralgia: determination of minimum clinically important difference in pain improvement for patient reported outcomes[J]. Neurosurgery.2013,72(5):749-754. 19.Hedayat AS,Wang J,Xu T. Minimum clinically important difference in medical studies[J]. important difference[J]. Journal of Central South University(Medical Science), 2009,34(11):1058-1062. 
21. Yang Z, Li W, Tu XM, Tang W, Messing S, Duan LP, Pan JH, Li XM, Wan CH., 2012. Validation and Psychometric Properties of Chinese Version of SF-36 in Patients with Hypertension, Coronary Heart Diseases, Chronic Gastritis and Peptic Ulcer. Int J Clin Pract 66(10):991-998.),

22. Wyrwich K, Tierney W, Wolinsky F. Using the standard error of measurement to identify important intra-individual change on the Asthma Quality of Life Questionnaire[J]. Qual Life Res 2002,11(1): 1 - 7.

23.. Jaeschke R, Singer J, Guyatt GH. Measurement of health status. Ascertaining the minimal clinically important difference. Control Clin Trials. 1989;10(4):407 - 415.

24. Stephen J. Walters, John E ,et al. Comparison of the minimally important difference for two health state utility measures: EQ-5D and SF-6D. [J] Quality of Life Research (2005)14: 1523 - 1532.

25.Lewandrowski K, Carvalho P, Carvalho, et al.Minimal Clinically Important Difference in Patient-Reported Outcome Measures with the Transforaminal Endoscopic Decompression for Lateral Recess and Foraminal Stenosis[J].Int J Spine Surg. 2020 ;14(2):254-266.

26.Jacquet C, Pioger C, Khakha R, et al..Evaluation of the "Minimal Clinically Important Difference" (MCID) of the KOOS, KSS and SF-12 scores after open-wedge high tibial osteotomy[J].Knee Surg Sports Traumatol Arthrosc. 2020 Apr 27.

27.Bae E, Choi SE, Lee H,et al.Validity of EQ-5D utility index and minimal clinically important difference estimation among patients with chronic obstructive pulmonary disease.[J]BMC Pulm Med, 2020 ;20(1):73. 28.Jones IA, Togashi R, Heckmann N,et al.Minimal clinically important difference (MCID) for patient-reported shoulder outcomes[J].J Shoulder Elbow Surg, 2020 Apr 2. pii: S1058-2746(20)30036-7.

29.Burgstaller JM, Wertli MM, Ulrich NH,et al.Evaluating the Minimal Clinically Important Difference of EQ-5D-3L in Patients with Degenerative Lumbar Spinal Stenosis: A Swiss Prospective Multi-Center Cohort Study.[J]Spine (Phila Pa 1976). 2020 ; doi: 10.1097/BRS.0000000000003501. 
348 30.Shulman MA, Kasza J, Myles PS.Defining the Minimal Clinically Important Difference and 349 Patient-acceptable Symptom State Score for Disability Assessment in Surgical Patients[J].Anesthesiology.

3502020 Mar 10. doi: 10.1097/ALN.000000000000324.

351 31.Toussaint A, Hüsing P, Gumz A,et al.Sensitivity to change and minimal clinically important difference 352 of the 7-item Generalized Anxiety Disorder Questionnaire (GAD-7)[J].J Affect Disord. 2020 Mar $353 \quad 15 ; 265: 395-401$. 